$1-1-1995$

\title{
Trace and Eigenvalue Inequalities of Ordinary and Hadamard Products for Positive Semidefinite Hermitian Matrices
}

\author{
Bo-Ying Wang \\ Beijing Normal University \\ Fuzhen Zhang \\ Nova Southeastern University, zhang@nova.edu
}

Follow this and additional works at: https://nsuworks.nova.edu/math_facarticles

Part of the Mathematics Commons

\section{NSUWorks Citation}

Wang, Bo-Ying and Zhang, Fuzhen, "Trace and Eigenvalue Inequalities of Ordinary and Hadamard Products for Positive Semidefinite Hermitian Matrices" (1995). Mathematics Faculty Articles. 130.

https://nsuworks.nova.edu/math_facarticles/130 


\title{
TRACE AND EIGENVALUE INEQUALITIES FOR ORDINARY AND HADAMARD PRODUCTS OF POSITIVE SEMIDEFINITE HERMITIAN MATRICES*
}

\author{
BO-YING WANG ${ }^{\dagger}$ AND FUZHEN ZHANG ${ }^{\ddagger}$
}

Dr. Zhang dedicates this paper to his mother who passed away on February 5, 1994.

Abstract. Let $A$ and $B$ be $n \times n$ positive semidefinite Hermitian matrices, let $\alpha$ and $\beta$ be real numbers, let $\circ$ denote the Hadamard product of matrices, and let $A_{k}$ denote any $k \times k$ principal submatrix of $A$. The following trace and eigenvalue inequalities are shown:

$$
\begin{gathered}
\operatorname{tr}(A \circ B)^{\alpha} \leq \operatorname{tr}\left(A^{\alpha} \circ B^{\alpha}\right), \quad \alpha \leq 0 \text { or } \alpha \geq 1, \\
\operatorname{tr}(A \circ B)^{\alpha} \geq \operatorname{tr}\left(A^{\alpha} \circ B^{\alpha}\right), \quad 0 \leq \alpha \leq 1, \\
\lambda^{1 / \alpha}\left(A^{\alpha} \circ B^{\alpha}\right) \leq \lambda^{1 / \beta}\left(A^{\beta} \circ B^{\beta}\right), \quad \alpha \leq \beta, \alpha \beta \neq 0, \\
\lambda^{1 / \alpha}\left[\left(A^{\alpha}\right)_{k}\right] \leq \lambda^{1 / \beta}\left[\left(A^{\beta}\right)_{k}\right], \quad \alpha \leq \beta, \alpha \beta \neq 0 .
\end{gathered}
$$

The equalities corresponding to the inequalities above and the known inequalities

$$
\operatorname{tr}(A B)^{\alpha} \leq \operatorname{tr}\left(A^{\alpha} B^{\alpha}\right), \quad|\alpha| \geq 1,
$$

and

$$
\operatorname{tr}(A B)^{\alpha} \geq \operatorname{tr}\left(A^{\alpha} B^{\alpha}\right), \quad|\alpha| \leq 1
$$

are thoroughly discussed. Some applications are given.

Key words. trace inequality, eigenvalue inequality, Hadamard product, Kronecker product, Schur-convex function, majorization

AMS subject classifications. 15A18, 15A39, 15A42, 15A45

1. Introduction. Let $A$ be an $n \times n$ complex matrix. We denote $\lambda(A)=$ $\left(\lambda_{1}(A), \ldots, \lambda_{n}(A)\right)$, where the $\lambda_{i}(A)$ 's are the eigenvalues of $A$; furthermore, we arrange $\lambda_{1}(A) \geq \cdots \geq \lambda_{n}(A)$ if they are all real. As usual, $A \circ B=\left(a_{i j} b_{i j}\right)$ is the Hadamard (entrywise or Schur) product of $A$ and $B$ when $A$ and $B$ are of the same size. For real vectors $x=\left(x_{1}, \ldots, x_{n}\right)$ and $y=\left(y_{1}, \ldots, y_{n}\right)$ with components in decreasing order, we write $x \leq y$ if $x_{i} \leq y_{i}, i=1, \ldots, n ; \quad x \prec_{w} y$ if $x$ is weakly majorized by $y$, i.e., $\sum_{i=1}^{k} x_{i} \leq \sum_{i=1}^{k} y_{i}, k=1, \ldots, n$; and $x \prec y$ if $x \prec_{w} y$ and $\sum_{i=1}^{n} x_{i}=\sum_{i=1}^{n} y_{i}$

For any scalar $\alpha$ and any $n \times n$ diagonalizable matrix $A$ with spectral decomposition $A=U D U^{*}$, where $D=\operatorname{diag}\left\{\lambda_{1}(A), \ldots, \lambda_{n}(A)\right\}$ and $U$ is unitary, we define (for more general definition, see [HJ, p. 411])

$$
A^{\alpha}=U D^{\alpha} U^{*}=U \operatorname{diag}\left\{\left(\lambda_{1}(A)\right)^{\alpha}, \ldots,\left(\lambda_{n}(A)\right)^{\alpha}\right\} U^{*}
$$

whenever all the $\left(\lambda_{i}(A)\right)^{\alpha}$ 's make sense, and denote

$$
\lambda^{\alpha}(A)=(\lambda(A))^{\alpha}=\lambda\left(A^{\alpha}\right) .
$$

* Received by the editors August 12, 1993; accepted for publication (in revised form) by R. Horn October 28, 1994.

$\dagger$ Department of Mathematics, Beijing Normal University, Beijing 100875, China. The work of this author was supported by a National Science Foundation grant of China.

$\ddagger$ Department of Mathematics, Nova Southeastern University, Fort Lauderdale, Florida 33314 (zhang@alpha.nova.edu). 
We write $A \geq 0$ if $A$ is a positive semidefinite Hermitian matrix, and $A \geq B$ if $A$ and $B$ are Hermitian and $A-B \geq 0$. Throughout this paper we assume that $A \geq 0$, $B \geq 0, \alpha$ and $\beta$ are positive numbers unless $A$ and $B$ are both positive definite, in which case $\alpha$ and $\beta$ can be any real numbers, and $m$ is a positive integer. It is well known [HH, Corollary 2.3] that the product of two positive semidefinite Hermitian matrices is diagonalizable and has nonnegative eigenvalues.

While studying the moments of the eigenvalues of Schrödinger Hamiltonians in quantum mechanics, Lieb and Thirring [LT] first showed (in the setting of operators on a separable Hilbert space) that

$$
\operatorname{tr}(A B)^{\alpha} \leq \operatorname{tr}\left(A^{\alpha} B^{\alpha}\right)
$$

for any real number $\alpha \geq 1$.

The inequalities in (1) were extended to unbounded operators by Araki [Ar]. Upper and lower bounds for $\operatorname{tr}(A B)^{m}$ and $\operatorname{tr}\left(A^{m} B^{m}\right)$ when $m$ is a positive integer were obtained by Marcus $[\mathrm{M}]$, Le Couteur $[\mathrm{C}]$, and proved again by Bushell and Trustrum [BT]:

$$
\sum_{i=1}^{n} \lambda_{i}^{m}(A) \lambda_{n-i+1}^{m}(B) \leq \operatorname{tr}(A B)^{m} \leq \operatorname{tr}\left(A^{m} B^{m}\right) \leq \sum_{i=1}^{n} \lambda_{i}^{m}(A) \lambda_{i}^{m}(B) .
$$

In a recent paper, Wang and Gong [WG] generalized the above results in terms of majorization, and proved

$$
\log \lambda^{1 / \alpha}\left(A^{\alpha} B^{\alpha}\right) \prec \log \lambda^{1 / \beta}\left(A^{\beta} B^{\beta}\right), \quad 0<\alpha<\beta,
$$

as consequences

$$
\begin{gathered}
\lambda^{1 / \alpha}\left(A^{\alpha} B^{\alpha}\right) \prec_{w} \lambda^{1 / \beta}\left(A^{\beta} B^{\beta}\right), \quad 0<\alpha \leq \beta, \\
\lambda^{1 / \beta}\left(A^{\beta} B^{\beta}\right) \prec_{w} \lambda^{1 / \alpha}\left(A^{\alpha} B^{\alpha}\right), \quad \alpha \leq \beta<0, \\
\lambda^{\alpha}(A B) \prec_{w} \lambda\left(A^{\alpha} B^{\alpha}\right), \quad|\alpha| \geq 1,
\end{gathered}
$$

and

$$
\lambda\left(A^{\alpha} B^{\alpha}\right) \prec_{w} \lambda^{\alpha}(A B), \quad|\alpha| \leq 1 .
$$

We are concerned with analogues of these inequalities for the entrywise product. A simple example shows that an analogue of (2)

$$
\sum_{i=1}^{n} \lambda_{i}^{m}(A) \lambda_{n-i+1}^{m}(B) \leq \operatorname{tr}(A \circ B)^{m}
$$

does not hold in general: take $A=\left(\begin{array}{ll}1 & 0 \\ 0 & 1\end{array}\right), B=\left(\begin{array}{ll}1 & 1 \\ 1 & 1\end{array}\right)$, and $m=2$. However, the inequality

$$
\operatorname{tr}\left(A^{m} \circ B^{m}\right) \leq \sum_{i=1}^{n} \lambda_{i}^{m}(A) \lambda_{i}^{m}(B)
$$


is valid, due to the majorization (see, e.g., [H, p. 146], [BS], or [Z])

$$
\lambda(A \circ B) \prec_{w} \lambda(A) \circ \lambda(B), \quad \text { whenever } A, B \geq 0 .
$$

Consequently, we always have

$$
\lambda\left(A^{m} \circ B^{m}\right) \prec_{w} \lambda\left(A^{m}\right) \circ \lambda\left(B^{m}\right) .
$$

It will be seen shortly that

$$
\lambda^{1 / \alpha}\left(A^{\alpha} \circ B^{\alpha}\right) \leq \lambda^{1 / \beta}\left(A^{\beta} \circ B^{\beta}\right)
$$

for any nonzero real numbers $\alpha$ and $\beta$ such that $\alpha \leq \beta$. In particular

$$
\lambda^{m}(A \circ B) \leq \lambda\left(A^{m} \circ B^{m}\right), \quad m=1,2, \ldots
$$

In this paper we first give necessary and sufficient conditions for equalities in (1), (5), and (6) to hold, then show some eigenvalue inequalities for principal submatrices and matrix powers. Finally we discuss an analogue of the Lieb-Thirring inequality (1) for the Hadamard product and present some applications.

2. Trace inequalities for ordinary product. This section is devoted to the discussion of the Lieb-Thirring inequality (1) and majorizations (5) and (6). Necessary and sufficient conditions for trace equalities to hold, i.e., for $\prec_{w}$ in (5) and (6) to become $\prec$, are given.

In the following (and thereafter), $A$ and $B$ are automatically understood to be positive definite when $\alpha$ (or $\beta$ ) is negative or equal to 0 .

THEOREM 2.1. Let $A$ and $B$ be positive semidefinite Hermitian matrices. Then

$$
\operatorname{tr}(A B)^{\alpha} \leq \operatorname{tr}\left(A^{\alpha} B^{\alpha}\right), \quad \text { whenever }|\alpha| \geq 1,
$$

and

$$
\operatorname{tr}(A B)^{\alpha} \geq \operatorname{tr}\left(A^{\alpha} B^{\alpha}\right), \quad \text { whenever }|\alpha| \leq 1 \text {. }
$$

Equality holds for some value of $\alpha$ if and only if $\alpha=-1,0,1$, or $A B=B A$.

Proof. The inequalities follow from (5) and (6) which have appeared in [WG]. We need consider only the equality case. Sufficiency is obvious if one recalls that $A$ and $B$ are simultaneously unitarily diagonalizable when $A$ and $B$ are normal and commute. To prove necessity, noticing that $\operatorname{tr}(A B)^{\alpha}=\operatorname{tr}\left(A^{-1} B^{-1}\right)^{-\alpha}$ when $\alpha<0$, we may assume that

$$
\operatorname{tr}(A B)^{\alpha}=\operatorname{tr}\left(A^{\alpha} B^{\alpha}\right), \text { for some } \alpha>0, \alpha \neq 1,
$$

and break down the proof into cases (a) $\alpha \geq 2$, (b) $1<\alpha<2$, and (c) $0<\alpha<1$. Equality holds trivially when $\alpha=0, A$ and $B$ are nonsingular.

(a) $\alpha \geq 2$. In this case we claim that $\operatorname{tr}(A B)^{\alpha}=\operatorname{tr}\left(A^{\alpha} B^{\alpha}\right)$ implies that $A B=B A$.

If $\alpha=2$, i.e., $\operatorname{tr}(A B)^{2}=\operatorname{tr}\left(A^{2} B^{2}\right)$, we assume, without loss of generality, that $A$ is a diagonal matrix with diagonal entries $a_{1}, \ldots, a_{n}$. Then

$$
\operatorname{tr}\left(A^{2} B^{2}\right)-\operatorname{tr}(A B)^{2}=\sum_{i, j} a_{i}^{2}\left|b_{i j}\right|^{2}-\sum_{i, j} a_{i} a_{j}\left|b_{i j}\right|^{2}=\sum_{i<j}\left(a_{i}-a_{j}\right)^{2}\left|b_{i j}\right|^{2}=0 .
$$

Thus $a_{i} b_{i j}=a_{j} b_{i j}$ for every pair of $i$ and $j$, i.e., $A B=B A$. 
For $\alpha>2$, we show that $\operatorname{tr}(A B)^{\alpha}=\operatorname{tr}\left(A^{\alpha} B^{\alpha}\right)$ implies $\operatorname{tr}(A B)^{2}=\operatorname{tr}\left(A^{2} B^{2}\right)$, which leads to $A B=B A$, as we have just seen.

If $\operatorname{tr}(A B)^{2} \neq \operatorname{tr}\left(A^{2} B^{2}\right)$, we apply the strictly increasing and strictly Schur-convex function (see [MO, p. 60, A.8.a]) $\sum_{i=1}^{n} t_{i}^{\alpha / 2}$ to the weak majorization $\lambda^{2}(A B) \prec_{w}$ $\lambda\left(A^{2} B^{2}\right)$, and get

$$
\operatorname{tr}(A B)^{\alpha}<\sum_{i=1}^{n} \lambda_{i}^{\alpha / 2}\left(A^{2} B^{2}\right) \leq \sum_{i=1}^{n} \lambda_{i}\left(A^{\alpha} B^{\alpha}\right)=\operatorname{tr}\left(A^{\alpha} B^{\alpha}\right),
$$

where the last inequality follows from (5), a contradiction.

(b) $1<\alpha<2$. In this case we claim that

$$
\operatorname{tr}(A B)^{x}=\operatorname{tr}\left(A^{x} B^{x}\right) \text { for all } 1<x<\alpha .
$$

In fact, if $\operatorname{tr}(A B)^{x_{0}} \neq \operatorname{tr}\left(A^{x_{0}} B^{x_{0}}\right)$ for some $x_{0}$ and $1<x_{0}<\alpha$, applying the strictly increasing and strictly Schur-convex function $\sum_{i=1}^{n} t_{i}^{\alpha / x_{0}}$ to $\lambda^{x_{0}}(A B) \prec_{w} \lambda\left(A^{x_{0}} B^{x_{0}}\right)$, where $\prec_{w}$ is strict, we have

$$
\begin{aligned}
\operatorname{tr}(A B)^{\alpha} & =\sum_{i=1}^{n} \lambda_{i}^{\alpha}(A B) \\
& <\sum_{i=1}^{n} \lambda_{i}^{\alpha / x_{0}}\left(A^{x_{0}} B^{x_{0}}\right) \\
& \leq \sum_{i=1}^{n} \lambda_{i}\left(A^{\alpha} B^{\alpha}\right), \quad \text { (use (5)) }
\end{aligned}
$$

a contradiction. Thus $\operatorname{tr}(A B)^{x}-\operatorname{tr}\left(A^{x} B^{x}\right)$ is identically zero for $1<x<\alpha$.

Now expanding $\operatorname{tr}(A B)^{x}-\operatorname{tr}\left(A^{x} B^{x}\right)$ as a series of $x$ and using the fact [Co, pp. 31, 78] that if a series converges to zero on an open interval, then it converges to zero on the whole real number line, we have $\operatorname{tr}(A B)^{x}-\operatorname{tr}\left(A^{x} B^{x}\right)=0$, that is, $\operatorname{tr}(A B)^{x}=\operatorname{tr}\left(A^{x} B^{x}\right)$ for all real $x>0$, particularly for 2, thus $A B=B A$.

(c) $0<\alpha<1$. We show that

$$
\operatorname{tr}(A B)^{x}=\operatorname{tr}\left(A^{x} B^{x}\right), \quad \text { for all } \alpha<x<1 .
$$

Otherwise, $\operatorname{tr}(A B)^{x_{0}}>\operatorname{tr}\left(A^{x_{0}} B^{x_{0}}\right)$ for some $x_{0}$ and $\alpha<x_{0}<1$. Applying the strictly increasing and strictly Schur-convex function $\sum_{i=1}^{n} e^{\frac{\alpha}{x_{0}} t_{i}}$ to $\log \lambda\left(A^{x_{0}} B^{x_{0}}\right) \prec$ $\log \lambda^{x_{0}}(A B)$ (see [WG, Theorem 6]) when both of $A$ and $B$ are nonsingular, we have

$$
\begin{aligned}
\operatorname{tr}\left(A^{\alpha} B^{\alpha}\right) & =\sum_{i=1}^{n} \lambda_{i}\left(A^{\alpha} B^{\alpha}\right) \\
& \left.\leq \sum_{i=1}^{n} \lambda_{i}^{\alpha / x_{0}}\left(A^{x_{0}} B^{x_{0}}\right) \quad \text { (use }(6)\right) \\
& <\sum_{i=1}^{n}\left[\lambda_{i}(A B)\right]^{\alpha} \\
& =\operatorname{tr}(A B)^{\alpha},
\end{aligned}
$$

a contradiction. Due to the same reason as in (b), $\operatorname{tr}(A B)^{x}=\operatorname{tr}\left(A^{x} B^{x}\right)$ for all real $x>0$, thus $A B=B A$ when $A$ and $B$ are nonsingular. The singular case can be accomplished by the usual technique of continuity. 
Notice that when two normal matrices commute they are simultaneously unitarily diagonalizable. The corollary below is immediate.

Corollary 2.2. Let $A$ and $B$ be positive semidefinite Hermitian matrices. If $\operatorname{tr}(A B)^{\alpha}=\operatorname{tr}\left(A^{\alpha} B^{\alpha}\right), \alpha \neq-1,0,1$, then $(A B)^{\alpha}=A^{\alpha} B^{\alpha}$.

3. Eigenvalue inequalities for principal submatrices. For an $n \times n$ matrix $A$, we use $A_{k}$ to designate any $k \times k$ principal submatrix of $A, 1 \leq k \leq n$. A result of Ando [A, Corollary 4.2] yields the following lemma when one notices that the map $A \rightarrow A_{k}$ is normalized positive linear (see [A] for the definition).

Lemma 3.1. Let $A$ be an $n \times n$ positive semidefinite Hermitian matrix. Then

$$
A_{k} \leq\left[\left(A^{\alpha}\right)_{k}\right]^{1 / \alpha}, \quad 1 \leq \alpha<\infty
$$

and

$$
A_{k} \geq\left[\left(A^{-\alpha}\right)_{k}\right]^{-1 / \alpha}, \quad 1 \leq \alpha<\infty
$$

The following theorem says that $\lambda^{1 / x}\left[\left(A^{x}\right)_{k}\right]$ is a monotone vector-valued function of $x$.

TheOREM 3.2. Let $A$ be an $n \times n$ positive semidefinite Hermitian matrix. Then

$$
\lambda^{1 / \alpha}\left[\left(A^{\alpha}\right)_{k}\right] \leq \lambda^{1 / \beta}\left[\left(A^{\beta}\right)_{k}\right], \quad \text { whenever } \alpha \leq \beta, \alpha \beta \neq 0,
$$

with equality if and only if $\alpha=\beta$ or $A=P\left(A_{k} \oplus H\right) P^{T}$ for some $H \geq 0$ and some permutation matrix $P$.

Proof. For $0<\alpha \leq 1$, using (10), we have

$$
\left(A^{\alpha}\right)_{k} \leq\left[\left(A^{\alpha \frac{1}{\alpha}}\right)_{k}\right]^{\alpha}=\left(A_{k}\right)^{\alpha} .
$$

For $-1 \leq \alpha<0$, using (11), we have

$$
\left(A^{\alpha}\right)_{k} \geq\left[\left(A^{\alpha \frac{1}{\alpha}}\right)_{k}\right]^{\alpha}=\left(A_{k}\right)^{\alpha} .
$$

Thus

$$
\left(A_{k}\right)^{\alpha} \geq\left(A^{\alpha}\right)_{k}, \quad 0<\alpha \leq 1
$$

and

$$
\left(A_{k}\right)^{\alpha} \leq\left(A^{\alpha}\right)_{k}, \quad-1 \leq \alpha<0 .
$$

For $\alpha \leq \beta$ with the same sign, using (13), we get

$$
\left(A^{\beta}\right)_{k}^{\alpha / \beta} \geq\left(A^{\alpha}\right)_{k}, \quad \text { when } 0<\alpha / \beta \leq 1,
$$

and

$$
\left(A^{\alpha}\right)_{k}^{\beta / \alpha} \geq\left(A^{\beta}\right)_{k}, \quad \text { when } 0<\beta / \alpha \leq 1
$$

in either case

$$
\lambda^{1 / \alpha}\left[\left(A^{\alpha}\right)_{k}\right] \leq \lambda^{1 / \beta}\left[\left(A^{\beta}\right)_{k}\right]
$$


If $\alpha \leq \beta$ with different signs, using (14),

$$
\left(A^{\alpha}\right)_{k}^{\beta / \alpha} \leq\left(A^{\beta}\right)_{k}, \quad \text { when }-1 \leq \beta / \alpha<0,
$$

and

$$
\left(A^{\beta}\right)_{k}^{\alpha / \beta} \leq\left(A^{\alpha}\right)_{k}, \quad \text { when }-1 \leq \alpha / \beta<0
$$

in either case we have

$$
\lambda^{1 / \alpha}\left[\left(A^{\alpha}\right)_{k}\right] \leq \lambda^{1 / \beta}\left[\left(A^{\beta}\right)_{k}\right]
$$

Thus inequality (12) follows immediately.

Now we discuss the equality case in (12). Without loss of generality, we may assume that $A_{k}$ lies in the upper-left corner of $A$, i.e., we partition $A$ as $A=\left(\begin{array}{cc}A_{k} & C \\ C^{*} & H\end{array}\right)$, where $H$ is some positive semidefinite Hermitian matrix. We first consider the case where $\alpha=1$ or $\beta=1$ and $\alpha \neq \beta$. Suppose

$$
\lambda\left(A_{k}\right)=\lambda^{1 / s}\left[\left(A^{s}\right)_{k}\right]
$$

for some $s \neq 0,1$. Then

$$
\lambda\left(A_{k}\right)=\lambda^{1 / x}\left[\left(A^{x}\right)_{k}\right]
$$

for all $x \neq 0$ between $s$ and 1 , because of (12). Thus we can always find an interval $I$ between $s$ and 1 on the positive real number line, such that

$$
\lambda^{x}\left(A_{k}\right)=\lambda\left(A^{x}\right)_{k}, \quad x \in I,
$$

that is,

$$
\operatorname{tr}\left(A_{k}\right)^{x}-\operatorname{tr}\left(A^{x}\right)_{k}=0, \quad x \in I,
$$

which is the same as

$$
\operatorname{tr}(B A)^{x}-\operatorname{tr}\left(B^{x} A^{x}\right)=0, \quad x \in I
$$

where $B=\left(\begin{array}{cc}I_{k} & 0 \\ 0 & 0\end{array}\right)$. Hence $A B=B A$ by Theorem 1 , which leads to $A=A_{k} \oplus H$ as required.

For general $\alpha$ and $\beta$ with $\alpha<\beta$ and $\alpha \beta \neq 0$, if

$$
\lambda^{1 / \alpha}\left[\left(A^{\alpha}\right)_{k}\right]=\lambda^{1 / \beta}\left[\left(A^{\beta}\right)_{k}\right]
$$

we rewrite it as

$$
\lambda\left[\left(A^{\alpha}\right)_{k}\right]=\lambda^{\alpha / \beta}\left[\left(A^{\beta}\right)_{k}\right]=\lambda^{\alpha / \beta}\left\{\left[\left(A^{\alpha}\right)^{\beta / \alpha}\right]_{k}\right\} .
$$

The earlier argument yields $A^{\alpha}=\left(A^{\alpha}\right)_{k} \oplus \tilde{H}$ for some $\tilde{H} \geq 0$. Thus

$$
A=\left(A^{\alpha}\right)^{1 / \alpha}=\left[\left(A^{\alpha}\right)_{k}\right]^{1 / \alpha} \oplus(\tilde{H})^{1 / \alpha}=A_{k} \oplus H,
$$

where $H=(\tilde{H})^{1 / \alpha}$. 
CoRollary 3.3. If $A$ is an $n \times n$ positive semidefinite Hermitian matrix, then

$$
\lambda^{\alpha}\left(A_{k}\right) \leq \lambda\left[\left(A^{\alpha}\right)_{k}\right], \quad \alpha \leq 0 \quad \text { or } \quad 1 \leq \alpha
$$

and

$$
\lambda^{\alpha}\left(A_{k}\right) \geq \lambda\left[\left(A^{\alpha}\right)_{k}\right], \quad 0<\alpha<1
$$

with equality if and only if $\alpha=0,1$, or $A=P\left(A_{k} \oplus H\right) P^{T}$.

Lemma 3.1 and Theorem 3.2 yield the following corollary.

Corollary 3.4. Let $A=\left(\begin{array}{ll}A_{1} & A_{2} \\ A_{2}^{*} & A_{3}\end{array}\right) \geq 0$ be an $n \times n$ matrix, and write $A^{\alpha}=\left(\begin{array}{ll}B_{1} & B_{2} \\ B_{2}^{*} & B_{3}\end{array}\right)$, where $A_{1}$ and $B_{1}$ are corresponding $k \times k$ principal submatrices of $A$ and $A^{\alpha}$, respectively. Then

$$
\begin{gathered}
A_{1} \leq B_{1}^{1 / \alpha}, \quad \alpha \geq 1, \\
A_{1}^{\alpha} \geq B_{1}, \quad 0<\alpha<1, \\
A_{1}^{\alpha} \leq B_{1}, \quad-1<\alpha<0, \\
A_{1} \geq B_{1}^{1 / \alpha}, \quad \alpha \leq-1 .
\end{gathered}
$$

Equality in each case holds if and only if one of the following conditions is satisfied:

1. $\alpha=1$

2. $\operatorname{tr} A_{1}^{\alpha}=\operatorname{tr} B_{1}$;

3. $A_{2}=B_{2}=0$, i.e., $A=A_{1} \oplus A_{3}$.

Moreover (2) and (3) are equivalent when $\alpha \neq 0,1$. Thus (2) is the same as $A_{1}^{\alpha}=B_{1}$ when $\alpha \neq 1$.

A direct computation gives the inequality $\left(A_{k}\right)^{2} \leq\left(A^{2}\right)_{k}$. However $\left(A_{k}\right)^{3} \leq\left(A^{3}\right)_{k}$ does not hold in general, as the following example shows.

Take $A$ to be the 4-by-4 matrix with (1,1)-entry 2 and 1 elsewhere, and $k=2$. Then $\left(A^{3}\right)_{2}-\left(A_{2}\right)^{3}=\left(\begin{array}{ll}16 & 14 \\ 14 & 12\end{array}\right)$, which is not positive semidefinite.

It is well known that $A \circ B$ is the principal submatrix of the Kronecker product $A \otimes B$ lying in the intersections of rows and columns $1, n+2, \ldots, n^{2}$ of $A \otimes B$. Considering $A \otimes B$ in Theorem 3.2 in place of $A$ and noticing that $(A \otimes B)^{t}=A^{t} \otimes B^{t}$ for any real number $t$, we have the following theorem.

THEOREM 3.5. Let $A$ and $B$ be positive semidefinite Hermitian matrices. Then

$$
\lambda^{1 / \alpha}\left(A^{\alpha} \circ B^{\alpha}\right) \leq \lambda^{1 / \beta}\left(A^{\beta} \circ B^{\beta}\right), \text { whenever } \alpha \leq \beta, \alpha \beta \neq 0 .
$$

It is immediate that for $A, B, \ldots, C$ positive semidefinite Hermitian matrices

$$
\lambda^{1 / \alpha}\left(A^{\alpha} \circ B^{\alpha} \circ \cdots \circ C^{\alpha}\right) \leq \lambda^{1 / \beta}\left(A^{\beta} \circ B^{\beta} \circ \cdots \circ C^{\beta}\right), \quad \alpha \leq \beta, \alpha \beta \neq 0 .
$$

Taking $\beta=1, \alpha=1$, and $\beta=1$ in Theorem 3.5, respectively, we get the following corollary.

Corollary 3.6. Let $A, B \geq 0$. Then

$$
\lambda^{\alpha}(A \circ B) \leq \lambda\left(A^{\alpha} \circ B^{\alpha}\right), \quad \alpha \leq 0 \text { or } \alpha \geq 1,
$$


and

$$
\lambda^{\alpha}(A \circ B) \geq \lambda\left(A^{\alpha} \circ B^{\alpha}\right), \quad 0<\alpha<1 \text {. }
$$

It is noted in $\S 4$ that equality holds in (15) or Corollary 3.6 if and only if $A$ and $B$ have the structures described in Theorem 4.1 or $\alpha=\beta \neq 0$ in (15), or if $\alpha=0,1$ in Corollary 3.6.

4. Trace inequalities for Hadamard product. The following is an analogue of Theorem 2.1 for the Hadamard product.

THEOREM 4.1. Let $A, B \geq 0$. Then for any real number $\alpha$

$$
\operatorname{tr}(A \circ B)^{\alpha} \leq \operatorname{tr}\left(A^{\alpha} \circ B^{\alpha}\right), \quad \text { if } \alpha \leq 0 \quad \text { or } 1 \leq \alpha,
$$

and

$$
\operatorname{tr}(A \circ B)^{\alpha} \geq \operatorname{tr}\left(A^{\alpha} \circ B^{\alpha}\right), \quad \text { if } 0<\alpha<1 .
$$

Equality occurs if and only if one of the following conditions is satisfied:

(i) $\alpha=0$ or 1 ;

(ii) $(A \circ B)^{\alpha}=A^{\alpha} \circ B^{\alpha}$;

(iii) there exists a permutation matrix $P$ such that

$$
A \otimes B=P[(A \circ B) \oplus H] P^{T}
$$

for some $H \geq 0$;

(iv) there exists a permutation matrix $P$ such that $P A P^{T}=D_{A} \oplus 0 \oplus \tilde{A}$ and $P B P^{T}=D_{B} \oplus \tilde{B} \oplus 0$, where $D_{A}$ and $D_{B}$ are invertible diagonal matrices of the same size, $\tilde{A}$ and $\tilde{B}$ are positive semidefinite Hermitian matrices each with the same size as 0 in the other direct sum;

(v) $(A \circ B)(X \circ Y)=(A X) \circ(B Y)$ for all $n \times m$ matrices $X$ and $Y$, where $m$ is an integer.

Moreover, (ii), (iii), (iv) and (v) are equivalent when $\alpha \neq 0,1$.

Proof. The trace inequalities (16) and (17) follow from Corollary 3.6. We need discuss only the equality case. We assume $\alpha \neq 0,1$, and show that "equality" $\Leftrightarrow($ ii $) \Leftrightarrow($ iii $) \Rightarrow$ (iv) $\Rightarrow$ (v), (iv) $\Rightarrow$ (ii), and (v) $\Rightarrow$ (iv).

Consider the Kronecker product $(A \otimes B)^{\alpha}=A^{\alpha} \otimes B^{\alpha}$ and note that $A^{\alpha} \circ B^{\alpha}$ is a principal submatrix of $A^{\alpha} \otimes B^{\alpha}$, consequently of $(A \otimes B)^{\alpha}$, lying in the same position as $A \circ B$ does in $A \otimes B$. If $\operatorname{tr}(A \circ B)^{\alpha}=\operatorname{tr}\left(A^{\alpha} \circ B^{\alpha}\right)$, then (ii), equivalently (iii), results from Corollary 3.4. To obtain (iv), we notice that for any permutation matrix $Q$

$$
\operatorname{tr} Q(A \circ B)^{\alpha} Q^{T}=\operatorname{tr} Q\left(A^{\alpha} \circ B^{\alpha}\right) Q^{T}
$$

and

$$
\operatorname{tr}\left(Q A Q^{T} \circ Q B Q^{T}\right)^{\alpha}=\operatorname{tr}\left(Q A Q^{T}\right)^{\alpha} \circ\left(Q B Q^{T}\right)^{\alpha} .
$$

Thus we may assume $b_{11} \neq 0$ if $B \neq 0$ and consider the first row of $A \otimes B=\left(a_{i j} B\right)$. $a_{11} b_{11}$ appears in $A \circ B$; for $j>1, a_{1 j} b_{11}$ lies on none of columns $1, n+2, \ldots, n^{2}$. In other words, if $R(A \otimes B) R^{T}=\left(\begin{array}{cc}A \circ B & A_{2} \\ A_{2}^{*} & A_{3}\end{array}\right)$ for some permutation matrix $R$, then 
$a_{1 j} b_{11}$ is contained in $A_{2}$. Applying Corollary 3.4 or by (iii), we have $A_{2}=0$. Hence $a_{1 j} b_{11}=0$, and $a_{1 j}=0=\overline{a_{j 1}}$ for $j>1$. Interchanging the roles of $A$ and $B$, we obtain $b_{1 j}=b_{j 1}=0$ for $j>1$ if $a_{11} \neq 0$. Repeating the argument for all $b_{i i} \neq 0$, we see that for some permutation matrix $S$

$$
S B S^{T}=\left(\begin{array}{ccccc}
b_{1}^{\prime} & * & \cdots & * & \\
* & \ddots & \ddots & \vdots & \\
\vdots & \ddots & \ddots & * & \\
* & \cdots & * & b_{k}^{\prime} & \\
& & & & 0_{n-k}
\end{array}\right)
$$

and

$$
S A S^{T}=\left(\begin{array}{ccccc}
a_{1}^{\prime} & 0 & \cdots & 0 & \\
0 & \ddots & \ddots & \vdots & \\
\vdots & \ddots & \ddots & 0 & \\
0 & \cdots & 0 & a_{k}^{\prime} & \\
& & & & \tilde{A}_{n-k}
\end{array}\right)
$$

where $b_{1}^{\prime}, \ldots, b_{k}^{\prime}$ are the nonzero $b_{i i}$ 's and $\tilde{A}_{n-k}$ is an $(n-k)$-square positive semidefinite Hermitian matrix. Let $a_{1}, \ldots, a_{s}$ be those of $a_{1}^{\prime}, \ldots, a_{k}^{\prime}$ which are nonzero, then we have a permutation matrix $P$ such that

$$
P A P^{T}=\left(\begin{array}{ccccc}
a_{1} & & 0 & & \\
& \ddots & & & \\
0 & & a_{s} & & \\
& & & 0_{t} & \\
& & & & \tilde{A}_{n-s-t}
\end{array}\right)
$$

and

$$
P B P^{T}=\left(\begin{array}{ccccc}
b_{1} & & 0 & & \\
& \ddots & & & \\
0 & & b_{s} & & \\
& & & \tilde{B}_{t} & \\
& & & & 0_{n-s-t}
\end{array}\right)
$$

where $b_{1}, \ldots, b_{s}$ are not equal to zero. (iv) follows. Thus we have proved the implications "equality" $\Leftrightarrow$ (ii) $\Leftrightarrow$ (iii) $\Rightarrow$ (iv).

Direct computations give (iv) $\Rightarrow$ (ii) and (iv) $\Rightarrow(\mathrm{v})$. To see (v) $\Rightarrow$ (iv), we take $X=A$ and $Y=B$ in (v). Then $(A \circ B)^{2}=A^{2} \circ B^{2}$ which results in (iv), as seen.

Going back to Theorem 3.5, we see equality in (15) holds, that is,

$$
\lambda\left(A^{\alpha} \circ B^{\alpha}\right)=\lambda^{\alpha / \beta}\left[\left(A^{\alpha}\right)^{\beta / \alpha} \circ\left(B^{\alpha}\right)^{\beta / \alpha}\right],
$$

if and only if either $\alpha=\beta \neq 0$ or $A$ and $B$ have the structures described in the previous theorem, by applying Theorem 4.1 to $A^{\alpha}$ and $B^{\alpha}$. 
5. Applications. The Lieb-Thirring inequality (1) may be investigated for a variety of real-valued matrix functions in the place of the trace function. We consider, as an example, the matrix function-sum of principal minors. Let $E_{k}(X)$ denote the sum of all the $\left(\begin{array}{c}n \\ k\end{array}\right) k$-square principal minors of the $n \times n$ matrix $X$, let $E_{k}(x)$ denote the $k$ th elementary symmetric function of the row vector $x$, and let $C_{k}(X)$ denote the $k$ th compound matrix of $X$. Then (see [MM, pp.18, 24])

$$
E_{k}(X)=\operatorname{tr} C_{k}(X)=E_{k}(\lambda(X)) .
$$

TheOREM 5.1. Let $A$ and $B$ be positive semidefinite Hermitian matrices. Then

$$
\begin{gathered}
E_{k}(A B)^{\alpha} \leq E_{k}\left(A^{\alpha} B^{\alpha}\right), \quad|\alpha| \geq 1, \\
E_{k}(A B)^{\alpha} \geq E_{k}\left(A^{\alpha} B^{\alpha}\right), \quad|\alpha| \leq 1, \\
E_{k}(A \circ B)^{\alpha} \leq E_{k}\left(A^{\alpha} \circ B^{\alpha}\right), \quad \alpha \leq 0 \quad \text { or } 1 \leq \alpha,
\end{gathered}
$$

and

$$
E_{k}(A \circ B)^{\alpha} \geq E_{k}\left(A^{\alpha} \circ B^{\alpha}\right), \quad 0 \leq \alpha \leq 1 .
$$

Equality holds in (19) or (20) if and only if $\alpha=-1,0,1$ or the kth compound matrices of $A$ and $B$ commute, and equality holds in (21) or (22) if and only if $\alpha=0,1$, the rank of $A^{\alpha} \circ B^{\alpha}$ is less than $k$, or $A$ and $B$ have the structures described in Theorem 4.1.

Proof. Noting that $\left.C_{k}(X Y)=C_{k}(X) C_{k}(Y)\right)$ and applying (18), we have for $|\alpha|>1$

$$
\begin{aligned}
E_{k}(A B)^{\alpha} & =\operatorname{tr} C_{k}(A B)^{\alpha} \\
& =\operatorname{tr}\left(C_{k}(A) C_{k}(B)\right)^{\alpha} \\
& \leq \operatorname{tr}\left(C_{k}(A)\right)^{\alpha}\left(C_{k}(B)\right)^{\alpha} \quad(\text { by Theorem 1) } \\
& =E_{k}\left(A^{\alpha} B^{\alpha}\right) .
\end{aligned}
$$

Equality holds if and only if $C_{k}(A) C_{k}(B)=C_{k}(B) C_{k}(A)$. The inequality is reversed when $|\alpha| \leq 1$.

For the case of the entrywise product and $\alpha \leq 0$ or $1 \leq \alpha$, we have

$$
\begin{aligned}
E_{k}(A \circ B)^{\alpha} & =E_{k}\left(\lambda^{\alpha}(A \circ B)\right) \\
& \leq E_{k}\left(\lambda\left(A^{\alpha} \circ B^{\alpha}\right)\right) \quad(\text { by Corollary } 3.3) \\
& =E_{k}\left(A^{\alpha} \circ B^{\alpha}\right) .
\end{aligned}
$$

Equality occurs if and only if either $\lambda^{\alpha}(A \circ B)=\lambda\left(A^{\alpha} \circ B^{\alpha}\right)$ or each term of $E_{k}\left(\lambda\left(A^{\alpha} \circ\right.\right.$ $\left.B^{\alpha}\right)$ ) vanishes. The former results in the structures of $A$ and $B$ given in Theorem 4.1 when $\alpha \neq 0,1$, and the latter is equivalent to $\lambda\left(A^{\alpha} \circ B^{\alpha}\right)$ containing at least $n-k+1$ zeros, that is, to $\operatorname{rank}\left(A^{\alpha} \circ B^{\alpha}\right)<k$. The case $0 \leq \alpha \leq 1$ is similarly discussed.

Remark 1. Theorems 2.1 and 4.1 are obtained if one takes $k=1$ in the previous theorem. If $k=n$, then (19) is the identity $\operatorname{det}(A B)^{\alpha}=\operatorname{det}\left(A^{\alpha} B^{\alpha}\right)$, and (21) 
becomes $\operatorname{det}(A \circ B)^{\alpha} \leq \operatorname{det}\left(A^{\alpha} \circ B^{\alpha}\right)$, both sides of which vanish when one of $A$ and $B$ is singular, since $\operatorname{rank}\left(A^{\alpha} \circ B^{\alpha}\right) \leq \operatorname{rank}\left(A^{\alpha} \otimes B^{\alpha}\right)=\operatorname{rank}(A) \operatorname{rank}(B)$.

Remark 2. Regarding Theorem 3.5, we can also prove, by using a result of Ando [A, Theorems 10 and 11], that for $A, B \geq 0$,

$$
\left(A^{\alpha} \circ B^{\alpha}\right)^{1 / \alpha} \leq\left(A^{\beta} \circ B^{\beta}\right)^{1 / \beta}, \quad \alpha \leq \beta \leq-1 \quad \text { or } \quad 1 \leq \alpha \leq \beta .
$$

The inequality above does not hold for all $\alpha \leq \beta, \alpha \beta \neq 0$, as the following example shows:

Take $\alpha=1 / 3, \beta=1, A=B=\left(\begin{array}{ll}2 & 1 \\ 1 & 1\end{array}\right)^{3}$. Then $\left(A^{1 / 3} \circ B^{1 / 3}\right)^{3} \not \subset A \circ B$, since

$$
\operatorname{det}\left[A \circ B-\left(A^{1 / 3} \circ B^{1 / 3}\right)^{3}\right]=\operatorname{det}\left[\left(\begin{array}{cc}
169 & 64 \\
64 & 25
\end{array}\right)-\left(\begin{array}{cc}
73 & 22 \\
22 & 7
\end{array}\right)\right]=-36<0 \text {. }
$$

In general, $(A \circ B)^{3} \not \leq A^{3} \circ B^{3}$. However, the inequality $(A \circ B)^{2} \leq A^{2} \circ B^{2}$ holds, as seen in $[\mathrm{A}],[\mathrm{H}]$, or $[\mathrm{Z}]$.

Acknowledgments. Dr. Zhang wishes to thank Professor E. H. Lieb for drawing his attention to $[\mathrm{Ar}]$ and Professor R. A. Horn and the referee for helpful suggestions and valuable comments.

\section{REFERENCES}

[A] T. ANDo, Concavity of certain maps on positive definite matrices and applications to Hadamard products, Linear Algebra Appl., 26(1979), pp. 203-241.

[Ar] H. ARAKI, On an inequality of Lieb and Thirring, Lett. in Math. Physics, 19(1990), pp. 167170.

[BS] R. B. BAPAT AND V. S. Sunder, On majorization and Schur products, Linear Algebra Appl., 72(1985), pp. 107-117.

[BT] P. J. Bushell and G. B. TRustrum, Trace inequalities for positive definite matrix power products, Linear Algebra Appl., 132(1990), pp. 173-178.

[C] K. J. LE CouteuR, Representation of the function $\operatorname{Tr}(\exp (A-\lambda B))$ as a Lapalace transform with positive weight and some matrix inequalities, J. Phys. A, 13(1980), pp. 3147-3159.

[Co] J. B. Conway, Functions of One Complex Variable, Second Edition, Springer-Verlag, New York, 1978.

[H] R. A. Horn, The Hadamard product, Proc. of Symposia in Applied Mathematics, Vol. 40, American Mathematical Society, Providence, 1990.

[HH] Y. HONG AND R. A. HORN, The Jordan canonical form of a product of a Hermitian and a positive semidefinite matrix, Linear Algebra Appl., 147(1991), pp. 373-386.

[HJ] R. A. HoRn AND C. R. JoHnson, Topics in Matrix Analysis, Cambridge University Press, New York, 1991.

[LT] E. H. Lieb AND W. Thirring, Studies in Mathematical Physics, Essays in Honor of Valentine Bartmann, Princeton University Press, Princeton, NJ, 1976.

[M] M. MARCUS, An eigenvalue inequality for the product of normal matrices, Amer. Math. Monthly, 63(1956), pp. 173-174.

[MM] M. Marcus AND H. Minc, A Survey of Matrix Theory and Matrix Inequalities, Dover Books, New York, 1992.

[MO] A. W. MARShall AND I. OlkIn, Inequalities: Theory of majorization and its applications, Academic Press, San Diego, CA, 1979.

[WG] B.-Y. WANG AND M.-P. GONG, Some eigenvalue inequalities for positive semidefinite matrix power products, Linear Algebra Appl., 184(1993), pp. 249-260.

[Z] F. ZHANG, Another proof of a singular value inequality concerning Hadamard products, Linear Multilinear Algebra, 22(1988), pp. 307-311. 
Reproduced with permission of the copyright owner. Further reproduction prohibited without permission. 